\title{
Research on Fuzzy Three Stage Tandem Queues
}

\author{
W. Ritha, J. Merline Vinotha, I. Antonitte Vinoline, Nivetha Martin
}

\begin{abstract}
This project intends a general procedure to obtain the membership function of the performance measures in three stage tandem queues when the inter-arrival time and service time are in fuzzy. The basic idea is to diminish the crisp queue into fuzzy by applying the a-cut approach technique. A pair of parametric program is formulated to depict that family of crisp queue in which the membership functions of the system performance are acquired.
\end{abstract}

Keywords- Fuzzy, queueing system, tandem queue, parametric programming, membership function.

\section{INTRODUCTION :}

Queueing theory is the mathematical diligence of waiting lines or queues. Queue lengths and waiting time can be signified by constructing the queueing model. The system in which single queues are affliated by a routing the network. Queueing theory plays an important role in our daily life. It is not possible to exactly determine the arrival and departure of customers when the number and types of facilities as well as the essential of the customers are not known. Queuing theory techniques, in particular, can help us to determine suitable number and type of service facilities to be provided to different types of customers. The applications of the queueing theory [4]are traffic flow, programming the patients in hospital, facility design in bank and other institutions,programming of service facilities in a repair and maintenance in workshop, programming of limited transport fleet to a large number of user,programming of reconstruction of used engines and assemblies of aircrafts, missile system, transport fleet.

The fuzzy queues are first proposed by R.J.Lie and E.S.Lee in 1989. In poisson arrival queuing system is a fairly reasonable appropriation where the arrival rate and service rate are really more possibilistic than probabilistic. But, in many practical situations the parameters $\lambda$ (arrival rate) and $\mu$ (service rate) are frequently fuzzy and cannot be expressed in exact terms.

\section{TANDEM QUEUE:}

Tandem queue is the simplest signal network of queue. The Jackson network is the first substantial result in the queue for which a proficient product from stationary distribution exists and the mean value interpretation which

Revised Manuscript Received on April 12, 2019.

W. Ritha, Department of Mathematics, Holy Cross College (Autonomous), Tiruchirapalli. T.N, India

J. Merline Vinotha, Department of Mathematics, Holy Cross College (Autonomous), Tiruchirapalli. T.N, India

I. Antonitte Vinoline, Department of Mathematics, Holy Cross College (Autonomous), Tiruchirapalli. T.N, India

Nivetha Martin, Department of Mathematics, Arul Anandar College (Autonomous), Karumathur. T.N, India concede average metrics such as throughput and sojourn times to be determined.

Tandem queue is modeled for multi-hop wireless network which is proposed by Long Bao Le, Erkam Hossain in 2007. The queue performs the QOS routing and admission control in the network. And also, tandem queue is modeled for coupled processor which is proposed by Jacques Resing, Lerzan ö Rmeci in 2003. This project analyse the two dimensional markov process represent the number of jobs in the two stations.

A customer server at point $i$ forthwith joins the queue at the point $i+1$, departing the system only when all the stages have been accomplished.

Many models have proposed tandem queue based upon the nature of fundamental process.

In this paper, we analyse the three stage tandem queues in fuzzy environment with trapezoidal fuzzy number.

\section{THREE STAGE TANDEM QUEUES:}

A Tandem queue with three service station $S_{1}, S_{2}, S_{3}$ with a poisson rate $\tilde{\lambda}$. The customers from outside enter into the station $S_{1}$, after service in $S_{1}$, moves to the station $S_{2}$, then they join the station $S_{3}$ in queue. After the termination of service at the station $S_{3}$, the customers can abandon the queueing system. The queue formation is infinite and service time of station $\mathrm{S}_{1}, \mathrm{~S}_{2}, \mathrm{~S}_{3}$ are exponentially distributed with parameters $\tilde{\mu}_{1}, \tilde{\mu}_{2}, \tilde{\mu}_{3}$ respectively. There must be an one customer is able to suffice of the service from each station at a time [6].

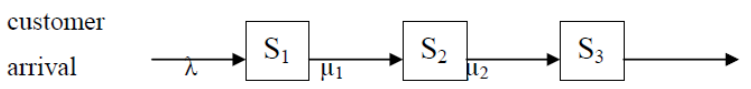

\section{PERFORMANCE MEASURE:}

1. The average number of customers in the queue.

$$
\mathrm{L}_{\mathrm{q}}=\lambda^{2}\left[\frac{1}{\mu_{1}\left(\mu_{1}-\lambda\right)}+\frac{1}{\mu_{2}\left(\mu_{2}-\lambda\right)}+\frac{1}{\mu_{3}\left(\mu_{3}-\lambda\right)}\right]
$$

2. The average waiting time of customer in the queue.

$$
\mathrm{W}_{\mathrm{q}}=\lambda\left[\frac{1}{\mu_{1}\left(\mu_{1}-\lambda\right)}+\frac{1}{\mu_{2}\left(\mu_{2}-\lambda\right)}+\frac{1}{\mu_{3}\left(\mu_{3}-\lambda\right)}\right]
$$

3. The average number of customers in the system.

$$
\mathrm{L}_{\mathrm{s}}=\frac{\lambda}{\mu_{1}-\lambda}+\frac{\lambda}{\mu_{2}-\lambda}+\frac{\lambda}{\mu_{3}-\lambda}
$$

4. The average waiting time of customer in the system.

Published By:

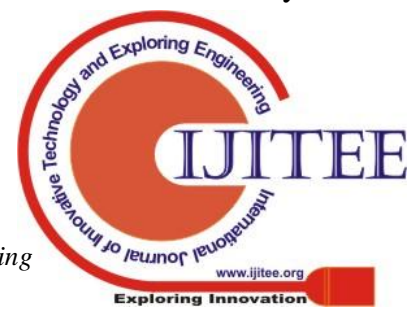




$$
\mathrm{W}_{\mathrm{s}}=\frac{1}{\mu_{1}-\lambda}+\frac{1}{\mu_{2}-\lambda}+\frac{1}{\mu_{3}-\lambda}
$$

\section{PROBLEM FORMULATION:}

STEP 1: When both inter-arrival time and service time are in fuzzy number, we use Zadeh's extension principle (using 1.11) to construct the membership function.

STEP 2: To derive the membership function, the beginning is to construct the $\alpha$-cuts of $\mu_{\mathrm{P}(\mathrm{A}, \mathrm{S})}$.

STEP 3: Then, we lack either $\mu_{\mathrm{A}}(\mathrm{a})=\alpha$ and $\mu_{\mathrm{S}}(\mathrm{s}) \geq \alpha$ or $\mu_{\mathrm{A}}(\mathrm{a}) \geq \alpha$ and $\mu_{\mathrm{S}}(\mathrm{s})=\alpha$ such that $\mathrm{z}=\mathrm{P}(\mathrm{A}, \mathrm{S})$ to satisfy $\mu_{\mathrm{P}(\mathrm{A}, \mathrm{S})}(\mathrm{z})=\alpha$.

The above steps obtained from the parametric programming technique [1].

The corresponding parametric programming for finding the lower and upper bounds of the $\alpha$-cut of $\mu_{\mathrm{P}(\mathrm{A}, \mathrm{S})}(\mathrm{z})$ are:

\section{FORMER CASE:}

$$
\begin{aligned}
& \mathrm{m}_{\mathrm{p}(\alpha)}^{\prime}=\min \mathrm{p}(\mathrm{a}, \mathrm{s}) \\
& \text { s.t. } \mathrm{m}_{\mathrm{A}(\alpha)} \leq \mathrm{a} \leq \mathrm{n}_{\mathrm{A}(\alpha)}, \mathrm{s} \in \mathrm{S}(\alpha) \\
& \mathrm{n}_{\mathrm{P}(\alpha)}^{\prime}=\max \mathrm{p}(\mathrm{a}, \mathrm{s}) \\
& \text { s.t. } \mathrm{m}_{\mathrm{A}(\alpha)} \leq \mathrm{a} \leq \mathrm{n}_{\mathrm{A}(\alpha)}, \mathrm{s} \in \mathrm{S}(\alpha)
\end{aligned}
$$

\section{LATTER CASE:}

$\mathrm{m}_{\mathrm{p}(\alpha)}^{\prime \prime}=\min \mathrm{p}(\mathrm{a}, \mathrm{s})$

s.t.m $\mathrm{S}_{\mathrm{S}(\alpha)} \leq \mathrm{s} \leq \mathrm{n}_{\mathrm{S}(\alpha)}, \mathrm{a} \in \mathrm{A}(\alpha)$

$\mathrm{n}_{\mathrm{P}(\alpha)}^{\prime \prime}=\max \mathrm{p}(\mathrm{a}, \mathrm{s})$

s.t. $\mathrm{m}_{\mathrm{S}(\alpha)} \leq \mathrm{s} \leq \mathrm{n}_{\mathrm{S}(\alpha)}, \mathrm{a} \in \mathrm{A}(\alpha)$

According to the definition 1.6 and $\mathrm{a} \in \mathrm{A}(\alpha), \mathrm{s} \in \mathrm{S}(\alpha)$ can be displaced by $\mathrm{a} \in\left[\mathrm{m}_{\mathrm{A}(\alpha)}, \mathrm{n}_{\mathrm{A}(\alpha)}\right]$ and $\mathrm{s} \in\left[\mathrm{m}_{\mathrm{S}(\alpha)}, \mathrm{n}_{\mathrm{S}(\alpha)}\right]$. Then equation (1),(2),(3),(4) can be written as in the form:

$$
\begin{aligned}
& \mathrm{m}_{\mathrm{p}(\alpha)}=\min \mathrm{p}(\mathrm{a}, \mathrm{s}) \\
& \text { s.t. } \mathrm{m}_{\mathrm{A}(\alpha)} \leq \mathrm{a} \leq \mathrm{n}_{\mathrm{A}(\alpha)} \text {, } \\
& \text { s.t. } \mathrm{m}_{\mathrm{S}(\alpha)} \leq \mathrm{s} \leq \mathrm{n}_{\mathrm{S}(\alpha)} \text {, }
\end{aligned}
$$

\section{UPPER BOUND:}

$\mathrm{n}_{\mathrm{P}(\alpha)}=\max \mathrm{p}(\mathrm{a}, \mathrm{s})$

s.t. $\mathrm{m}_{\mathrm{A}(\alpha)} \leq \mathrm{a} \leq \mathrm{n}_{\mathrm{A}(\alpha)}$,

s.t. $\mathrm{m}_{\mathrm{S}(\alpha)} \leq \mathrm{S} \leq \mathrm{n}_{\mathrm{S}(\alpha)}$,

since, $\left\{\left[\mathrm{m}_{\mathrm{p}(\alpha)}, \mathrm{n}_{\mathrm{p}(\alpha)}\right] / \alpha \in[0,1]\right\}$.

The membership function $\mu_{\mathrm{P}(\mathrm{A}, \mathrm{S})}(\mathrm{z})$ can be derived by using left shape function $\mathrm{m}_{\mathrm{p}(\alpha)}=\mathrm{L}(\mathrm{z})$ and right shape function $\mathrm{n}_{\mathrm{p}(\alpha)}=\mathrm{R}(\mathrm{z})$.

$$
\mu_{\mathrm{P}(\mathrm{A}, \mathrm{S})}(\mathrm{z})=\left\{\begin{array}{cc}
\mathrm{L}(\mathrm{z}), & \mathrm{q}_{1} \leq \mathrm{z} \leq \mathrm{q}_{2} \\
1, & \mathrm{q}_{2} \leq \mathrm{z} \leq \mathrm{q}_{3} \\
\mathrm{R}(\mathrm{z}), & \mathrm{q}_{3} \leq \mathrm{z} \leq \mathrm{q}_{4}
\end{array}\right.
$$

Where $\mathrm{q}_{1} \leq \mathrm{q}_{2} \leq \mathrm{q}_{3} \leq \mathrm{q}_{4}$ and $\mathrm{L}\left(\mathrm{q}_{1}\right)=\mathrm{R}\left(\mathrm{q}_{4}\right)=0$. Otherwise, $\alpha$ cut lies in the nested system.

\section{NUMERICAL EXAMPLE \& RESULTS}

Consider the three stage tandem queue where the interarrival time and service time are represented in fuzzy by $\tilde{\lambda}=[8,9,10,14], \quad \tilde{\mu}_{1}=[10,12,13,24], \quad \tilde{\mu}_{2}=[10,13,15,30]$, $\tilde{\mu}_{3}=[15,16,18,25]$.

The corresponding $\alpha$-cuts in the interval are:

$$
\begin{gathered}
{\left[\mathrm{m}_{\mathrm{A}(\alpha)}, \mathrm{n}_{\mathrm{A}(\alpha)}\right]=[\alpha+8,14-4 \alpha]} \\
{\left[\mathrm{m}_{\mathrm{S} 1(\alpha)}, \mathrm{n}_{\mathrm{S} 1(\alpha)}\right]=[2 \alpha+10,24-11 \alpha]} \\
{\left[\mathrm{m}_{\mathrm{S} 2(\alpha)}, \mathrm{n}_{\mathrm{S} 2(\alpha)}\right]=[3 \alpha+10,30-15 \alpha]} \\
{\left[\mathrm{m}_{\mathrm{S} 3(\alpha)}, \mathrm{n}_{\mathrm{S} 3(\alpha)}\right]=[\alpha+15,25-7 \alpha]}
\end{gathered}
$$

The parametric programs to be solved are:

$$
\mathrm{m}_{\mathrm{L}(\alpha)}=\min \left(\mathrm{a}^{2}\left[\frac{1}{\mathrm{~s}_{1}\left(\mathrm{~s}_{1}-\mathrm{a}\right)}+\frac{1}{\mathrm{~s}_{2}\left(\mathrm{~s}_{2}-\mathrm{a}\right)}+\frac{1}{\mathrm{~s}_{3}\left(\mathrm{~s}_{3}-\mathrm{a}\right)}\right]\right)
$$

subject to,

$$
\begin{gathered}
\alpha+8 \leq \mathrm{a} \leq 14-4 \alpha \\
2 \alpha+10 \leq \mathrm{s}_{1} \leq 24-11 \alpha \\
3 \alpha+10 \leq \mathrm{s}_{2} \leq 30-15 \alpha \\
\alpha+15 \leq \mathrm{s}_{3} \leq 25-7 \alpha \\
\mathrm{n}_{\mathrm{L}(\alpha)}=\max \left(\mathrm{a}^{2}\left[\frac{1}{\mathrm{~s}_{1}\left(\mathrm{~s}_{1}-\mathrm{a}\right)}+\frac{1}{\mathrm{~s}_{2}\left(\mathrm{~s}_{2}-\mathrm{a}\right)}+\frac{1}{\mathrm{~s}_{3}\left(\mathrm{~s}_{3}-\mathrm{a}\right)}\right]\right)
\end{gathered}
$$

subject to,

$$
\begin{gathered}
\alpha+8 \leq \mathrm{a} \leq 14-4 \alpha \\
2 \alpha+10 \leq \mathrm{s}_{1} \leq 24-11 \alpha \\
3 \alpha+10 \leq \mathrm{s}_{2} \leq 30-15 \alpha \\
\alpha+15 \leq \mathrm{s}_{3} \leq 25-7 \alpha
\end{gathered}
$$

To minimize the performance measure, a reaches its lower bound and $s_{1}, s_{2}, s_{3}$ reaches its upper bound then, $\mathrm{m}_{\mathrm{L}(\alpha)}=(8+\alpha)^{2}\left[\frac{1}{(24-11 \omega)(16-12 \alpha)}+\frac{1}{(30-15 \omega)(2416 \omega)}+\frac{1}{(25-7 \omega)(17-8 \alpha)}\right]$ and to maximize the performance measure, a reaches its upper bound and $s_{1}$, $\mathrm{s}_{2}, \mathrm{~s}_{3}$ reaches its lower bound.

$$
\mathrm{n}_{\mathrm{L}(\alpha)}=(14-4 \alpha)^{2}\left[\frac{1}{2(2 \alpha+1)(6 \alpha-4)}+\frac{1}{(3 \alpha+10)(7 \alpha-4)}+\frac{1}{(\alpha+15)(5 \alpha+1)}\right]
$$

The inverse function of $\mathrm{m}_{\mathrm{L}(\alpha)}$ and $\mathrm{n}_{\mathrm{L}(\alpha)}$ exist. Then, we can obtained the membership function $\mu_{\mathrm{L}}(\mathrm{z})$ as:

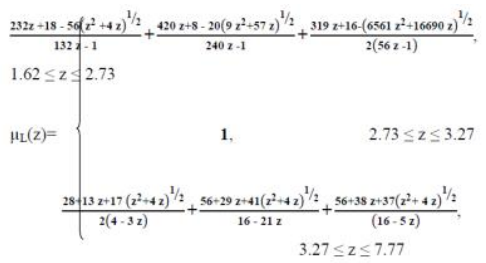


The membership function for $\mathrm{Wq}$ as follows:

The parametric programs to be solved are:

$$
\mathrm{m}_{\mathrm{W}(\alpha)}=\min \left(\mathrm{a}\left[\frac{1}{\mathrm{~s}_{1}\left(\mathrm{~s}_{1}-\mathrm{a}\right)}+\frac{1}{\mathrm{~s}_{2}\left(\mathrm{~s}_{2}-\mathrm{a}\right)}+\frac{1}{\mathrm{~s}_{3}\left(\mathrm{~s}_{3}-\mathrm{a}\right)}\right]\right)
$$

subject to,

$$
\begin{gathered}
\alpha+8 \leq \mathrm{a} \leq 14-4 \alpha \\
2 \alpha+10 \leq \mathrm{s}_{1} \leq 24-11 \alpha \\
3 \alpha+10 \leq \mathrm{s}_{2} \leq 30-15 \alpha \\
\alpha+15 \leq \mathrm{s}_{3} \leq 25-7 \alpha \\
\mathrm{n}_{\mathrm{W}(\alpha)}=\max \left(\mathrm{a}\left[\frac{1}{\mathrm{~s}_{1}\left(\mathrm{~s}_{1}-\mathrm{a}\right)}+\frac{1}{\mathrm{~s}_{2}\left(\mathrm{~s}_{2}-\mathrm{a}\right)}+\frac{1}{\mathrm{~s}_{3}\left(\mathrm{~s}_{3}-\mathrm{a}\right)}\right]\right)
\end{gathered}
$$

subject to,

$$
\begin{gathered}
\alpha+8 \leq \mathrm{a} \leq 14-4 \alpha \\
2 \alpha+10 \leq \mathrm{s}_{1} \leq 24-11 \alpha \\
3 \alpha+10 \leq \mathrm{s}_{2} \leq 30-15 \alpha \\
\alpha+15 \leq \mathrm{s}_{3} \leq 25-7 \alpha
\end{gathered}
$$

To minimize the performance measure, a reaches its lower bound and $s_{1}, s_{2}, s_{3}$ reaches its upper bound then, $\mathrm{m}_{\mathrm{W}(\alpha)}=(8+\alpha)\left[\frac{1}{(24-11 \alpha)(16-12 \alpha)}^{+} \frac{1}{(30-15 \alpha)(24-16 \alpha)}^{+} \frac{1}{(25-7 \alpha)(17-8 \alpha)}\right]$ and to maximize the performance measure, a reaches its upper bound and $s_{1}$, $\mathrm{s}_{2}, \mathrm{~s}_{3}$ reaches its lower bound.

$$
\mathrm{n}_{\mathrm{W}(\alpha)}=(14-4 \alpha)\left[\frac{1}{(2 \alpha+10)(6 \alpha-4)}+\frac{1}{(3 \alpha+10)(7 \alpha-4)}+\frac{1}{(\alpha+15)(5 a+1)}\right]
$$

The inverse function of $\mathrm{m}_{\mathrm{W}(\alpha)}$ and $\mathrm{n}_{\mathrm{W}(\alpha)}$ exist. Then, we can obtained the membership function as:

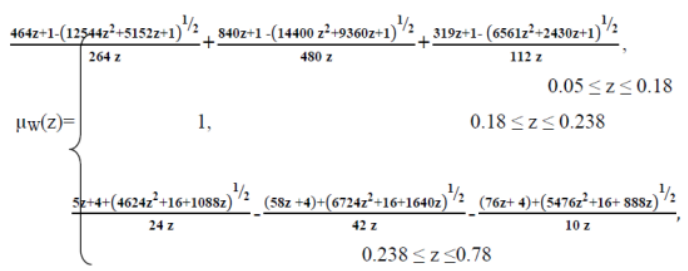

The membership function for Ls as follows:

The parametric programs to be solyed are: subject to,

$$
\left[\mathrm{m}_{\mathrm{L}(\alpha)}=\min \quad \mathrm{a}\left[\frac{1}{s_{1}-\mathrm{a}}+\frac{1}{s_{2}-\alpha}+\frac{1}{s_{3}-\mathrm{a}}\right]\right.
$$

$$
\begin{gathered}
\alpha+8 \leq \mathrm{a} \leq 14-4 \alpha \\
2 \alpha+10 \leq \mathrm{s}_{1} \leq 24-11 \alpha \\
3 \alpha+10 \leq \mathrm{s}_{2} \leq 30-15 \alpha \\
\alpha+15 \leq \mathrm{s}_{3} \leq 25-7 \alpha \\
\mathrm{n}_{\mathrm{L}(\alpha)}=\max a\left[\frac{1}{\mathrm{~s}_{1-\mathrm{a}}}+\frac{1}{\mathrm{~s}_{2}-\mathrm{a}}+\frac{1}{\mathrm{~s}_{3-\mathrm{a}}}\right]
\end{gathered}
$$

subject to,

$$
\begin{gathered}
\alpha+8 \leq \mathrm{a} \leq 14-4 \alpha \\
2 \alpha+10 \leq \mathrm{s}_{1} \leq 24-11 \alpha \\
3 \alpha+10 \leq \mathrm{s}_{2} \leq 30-15 \alpha
\end{gathered}
$$

$$
\alpha+15 \leq s_{3} \leq 25-7 \alpha
$$

To minimize the performance measure, a reaches its lower bound and $s_{1}, s_{2}, s_{3}$ reaches its upper bound then, $\mathrm{m}_{\mathrm{L}(\alpha)}=(8+\alpha)\left[\frac{1}{(16-12 \alpha)}^{+} \frac{1}{(24-16 \alpha)}^{+} \frac{1}{(17-8 \alpha)}\right]$ and to maximize the performance measure, a reaches its upper bound and $s_{1}, s_{2}$, $\mathrm{s}_{3}$ reaches its lower bound.

$$
\mathrm{n}_{\mathrm{L}(\alpha)}=(14-4 \alpha)\left[\frac{1}{(6 a-4)}^{+} \frac{1}{(7 a-4)}^{+} \frac{1}{(5 a+1)}\right]
$$

The inverse function of $\mathrm{m}_{\mathrm{L}(\alpha)}$ and $\mathrm{n}_{\mathrm{L}(\alpha)}$ exist. Then, we can obtained the membership function as:

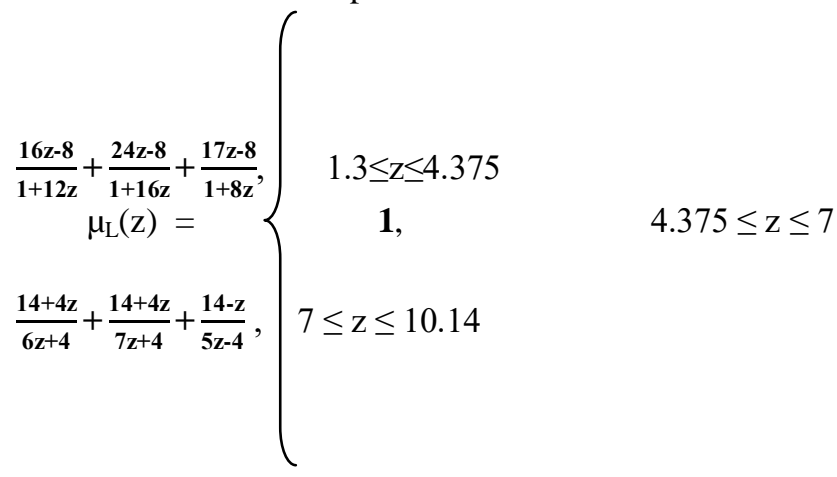

The membership function for Ws as follows:

The parametric programs to be solved are:

$$
\mathrm{m}_{\mathrm{W}(\alpha)}=\min \left(\frac{1}{\mathrm{~s}_{1}-\mathrm{a}}+\frac{1}{\mathrm{~s}_{2}-\mathrm{a}}+\frac{1}{\mathrm{~s}_{3}-\mathrm{a}}\right)
$$

subject to,

$$
\begin{gathered}
\alpha+8 \leq \mathrm{a} \leq 14-4 \alpha \\
2 \alpha+10 \leq \mathrm{s}_{1} \leq 24-11 \alpha \\
3 \alpha+10 \leq \mathrm{s}_{2} \leq 30-15 \alpha \\
\alpha+15 \leq \mathrm{s}_{3} \leq 25-7 \alpha \\
\mathrm{n}_{\mathrm{W}(\alpha)}=\max \left(\frac{1}{\mathrm{~s}_{1-\mathrm{a}}}+\frac{1}{\mathrm{~s}_{2}-\mathrm{a}}+\frac{1}{\mathrm{~s}_{3}-\mathrm{a}}\right)
\end{gathered}
$$

subject to

$$
\begin{gathered}
\alpha+8 \leq \mathrm{a} \leq 14-4 \alpha \\
2 \alpha+10 \leq \mathrm{s}_{1} \leq 24-11 \alpha \\
3 \alpha+10 \leq \mathrm{s}_{2} \leq 30-15 \alpha \\
\alpha+15 \leq \mathrm{s}_{3} \leq 25-7 \alpha
\end{gathered}
$$

To minimize the performance measure, a reaches its lower bound and $s_{1}, s_{2}, s_{3}$ reaches its upper bound then, $\mathrm{m}_{\mathrm{W}(\alpha)}=\left[\frac{1}{(16-12 \alpha)}+\frac{1}{(24-16 \alpha)}^{+} \frac{1}{(17-8 \alpha)}\right]$ and to maximize the performance measure, a reaches its upper bound and $s_{1}, s_{2}, s_{3}$ reaches its lower bound.

$$
\mathrm{n}_{\mathrm{W}(\alpha)}=\left[\frac{1}{(6 a-4)}+\frac{1}{(7 a-4)}+\frac{1}{(5 \alpha+1)}\right]
$$

The inverse function of $\mathrm{m}_{\mathrm{W}(\alpha)}$ and $\mathrm{n}_{\mathrm{W}(\alpha)}$ exist. Then, we can obtained the membership function as: 
$\mu_{\mathrm{W}}(\mathrm{z})=\mathbf{1}, \begin{cases}\frac{\mathbf{2 3 8} \mathrm{z}-\mathbf{5}}{\mathbf{4 8 z}}, & 0.163 \leq \mathrm{z} \leq 0.486 \\ \frac{\mathbf{2 1 8} \mathrm{z}+\mathbf{1 0 7}}{\mathbf{2 1 0 z}} & 0.486 \leq \mathrm{z} \leq 0.5 \\ & 0.5 \leq \mathrm{z} \leq 1\end{cases}$

\section{CONCLUSION:}

In this project, the analysis of fuzzy three stage tandem queues has been done when the inter-arrival time and service time are in fuzzy.We derive the membership function for the performance measure of three stage tandem queue by using the parametric programming technique which is based on $\alpha$-cut method, zadeh extension principle, right shape function and left shape function.

\section{REFERENCE:}

1. Chiang Kao, Chang-Chung Li, Shih-Pin Chen, Parametric programming to the analysis of fuzzy queues, Fuzzy sets and systems 107 (1), 93-100, 1999

2. D. Gross, C.M. Harris, Fundamentals of Queueing Theory, Wiley, New York, 1985.

3. R.J. Li, E.S. Lee, Analysis of fuzzy queues, Comput. Math. Appl. 17 (1989) 1143-1147.

4. S.Shanmugasundaram, P.Umarani, Queuing theory applied in our day to day life, 2015.

5. Sreekanth. K, Kamlesh Kumar, Performance Analysis of Three Stage Tandem Queues, jammu 2018.

6. Zimmermann, H. J., 2001. Fuzzy Set Theory and its Applications, fourth ed. Kluwer-Nijhoff, Boston. 